\title{
4 Health-IT Transformation im Kontext der Medizin und Public Health
}

\author{
Mustapha Addam
}

\section{Bestandsaufnahme und Stellenwert}

E-Health bzw. Health IT beschreibt ein abstraktes Verständnis über den Einsatz von Informations- und Kommunikationstechnologien im Gesundheitswesen. Ein einheitliches Verständnis ist in diesem Zusammenhang schwierig zu erfassen. Betrachtet man die Informationstechnologien im Gesundheitswesen im Allgemeinen, so lassen sich diese nach den übergreifenden Bereichen der Pathogenese und Salutogenese einteilen (s. Abb. 1). Dabei siedeln sich im medizinischen Bereich jene IT-Lösungen an, die hauptsächlich im ambulanten, stationären, rehabilitativen und pflegerischen Bereichen eingesetzt werden. Hierzu zählen ebenfalls Softwarelösungen, die durch Krankenkassen oder durch den Staat betrieben werden. Solche Softwarelösungen sind grundsätzlich für die Dokumentation, Verwaltung und Steuerung der gesamten Behandlungsabläufe von Patienten zuständig. Charakteristisch für diese Softwarelösungen, ist der indirekte Bezug des Patienten. Es existieren hierbei kaum bis gar keine Berührungspunkte zum Patienten. Die einzigen Berührungspunkte sind eine rudimentäre Einsicht in die Daten, z.B. Befunde, Entlassbriefe in Papierform oder eine Patienten-CD mit Bilddaten. Der Patient kann prinzipiell von seinen Rechten (Selbstbestimmungsrecht, Datenschutzrecht u.a. gesetzliche Grundlagen) zur Einsicht in die gespeicherte Patientendokumentation Gebrauch machen, dies erfolgt jedoch nach wie vor partiell in Papierform. Die Hoheit über diese IT-Lösungen obliegt dem medizinischen und pflegerischen Personal in den jeweiligen Einrichtungen des Gesundheitswesens. 


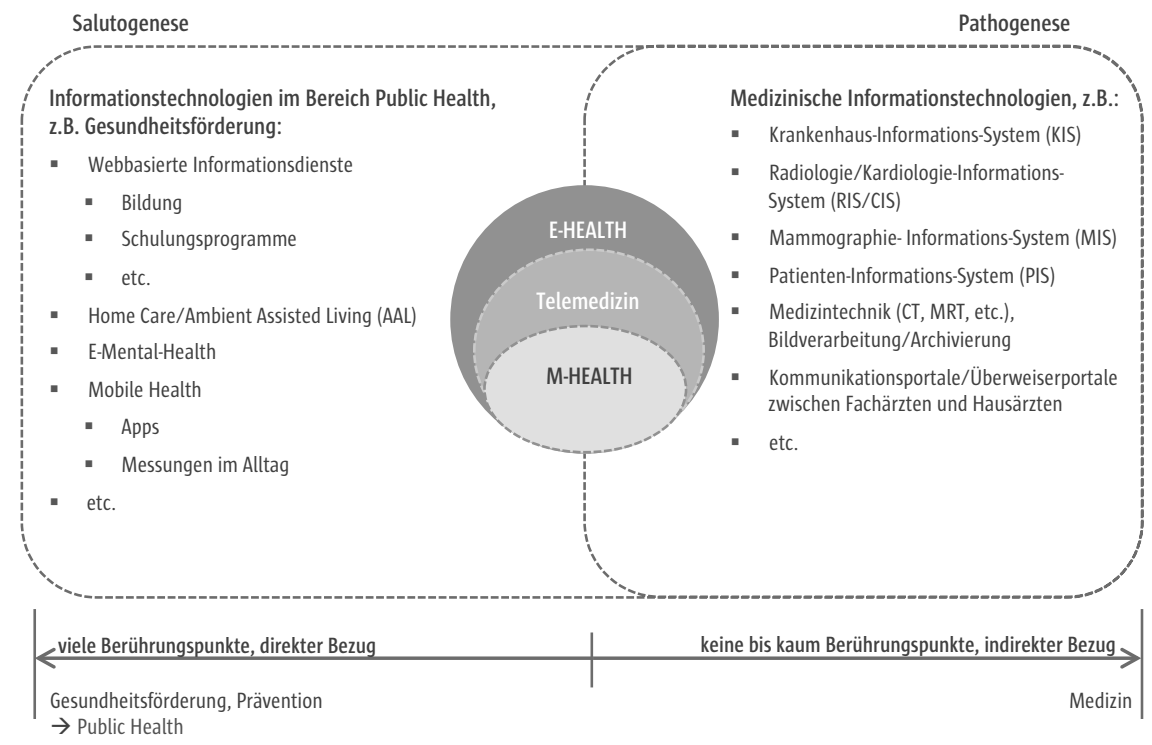

\section{Abb. 1 Health IT Abgrenzung nach den Bereichen Medizin und Public Health (eigene Darstellung)}

Anders als in der Medizin, umfasst der Public Health Bereich sämtliche Informationstechnologien die über den medizinischen Bereich hinausgehen. Kennzeichnend für diese Lösungen ist der direkte Bezug zum Menschen/Patienten. Direkter Bezug meint, jedes Individuum wird selbst aktiv und kümmert sich selbst um die eigenen Gesundheitsbelange. Solche Anwendungsszenarien können beispielsweise sein:

- Einholung von allgemeinen medizinischen Informationen über Onlineportale (Stichwort Google-Patient),

- Teilnahme an Online-Schulungsprogrammen z.B. in den Bereichen der Gesundheitsförderung, Betriebliche Gesundheitsförderung, E-Mental-Health u.a. Bereiche,

- Nutzung von mobilen Anwendungen zur Überwachung des eigenen Gesundheitszustandes und

- Einsatz von telemedizinischen Anwendungen zur Fernüberwachung der Patienten etc.

Im Bereich der Public Health obliegt die Hoheit über die Verwaltung der IT-Lösungen und Auswertung der Daten dem Individuum selbst.

\section{Beschreibung des Transformationsprozesses}

Im medizinischen Bereich sind die gängigen Arbeitsprozesse innerhalb von Gesundheitseinrichtungen von der Patientenaufnahme bis zur Entlassung und Nachsorge weitestgehend digitalisiert und standardisiert. Sicherlich gibt es in dieser Hinsicht viel Optimierungsbedarf, dennoch ist im Großen und Ganzen jede Gesundheitsein- 
richtung mit mindestens einem patientenführenden Informationssystem (KIS, RIS, LIS, AIS etc.) ausgestattet, das im Rahmen klinischer Entscheidungsprozesse unterstützt. Weiterhin ersetzen die integrierten Bildmanagementlösungen (z.B. PACS) nahezu vollständig filmbasierte Bilder und bilden damit elektronische Archive ab. Es lässt sich aktuell feststellen, dass der Markt für diese Systeme hinsichtlich der Marktabdeckung weitgehend gesättigt ist. In diesen Bereichen ist ein agiler bzw. disruptiver Verdrängungsmarkt schwer denkbar. Denn ist ein Informationssystem in einer Einrichtung bereits installiert und etabliert, so ist es schwierig aufgrund der Umstellungsaufwände, dieses durch ein anderes zu ersetzen. In Deutschland existiert eine Vielzahl an medizinischen Softwarelösungen, die in den Gesundheitseinrichtungen etabliert sind. In diesen Bereichen ist es für neue Unternehmungen schwierig, sich gegen die etablierten Anbieter zu behaupten. Für neue Start-ups ist eine Kooperation mit diesen Anbietern, im Besonderen in punkto der Realisierung von Schnittstellen mit den Herstellern administrativer Informationssysteme, nahezu unumgänglich. Sicherlich sind im medizinischen Bereich die Schnittstellen standardisiert und harmonisiert (z.B. DICOM, HL7, IHE etc.) und die Spezifikationen frei zugänglich, dennoch kann dieser Aspekt eine kostspielige Angelegenheit und damit ein Hemmnis sein. Zudem müssen Start-ups im medizinischen Bereich über einen langen Zeitraum bestimmte Gesetze, Regularien, Bestimmungen, Verordnungen etc., die sich beispielsweise aus den Medizinproduktegesetzen, Datenschutzgesetzen u.a. ergeben, umsetzen und überwinden. Diese Aspekte verzeichnen eher einen tradierten Entwicklungsverlauf. Äquivalente Entwicklungssprünge in Anlehnung anderer wirtschaftsbezogener Branchen sind im medizinischen Bereich aus den zuvor genannten Gründen schwerer vorstellbar. Denkbare Entwicklungssprünge wären beispielsweise im medizinischen Bereich die Konsolidierung der Health-IT-Märkte im Bereich der medizinischen Informationssysteme. Technologisch existiert, unabhängig von der Konsolidierung der Märkte, eine Vielzahl unbehandelter bzw. optimierungsbedürftiger Themengebiete, die Potenziale für disruptive Sprünge aufzeigen. Die Entwicklung ganzheitlicher elektronischer Patientenakten (EPA) läuft langsam voran. In dieser Hinsicht werden die Daten nach wie vor dezentral einrichtungsbzw. maximal verbundbezogen gespeichert, ein zentralisierter Zugriff auf Patientendaten ist kaum gegeben. Darüber hinaus sind die Themen der integrierten Versorgung, mit den Aspekten der intersektoralen und interdisziplinären Kommunikation bisher wenig ausgeprägt. Im Zeitalter der sehr weit fortgeschrittenen Technisierung und Digitalisierung geraten Patienten nach wie vor in lebensbedrohliche Situationen aufgrund verspätet zugestellter Patienteninformationen (z.B. Befunde etc.), die auf manuellen Wegen (z.B. Fax, Eilversand, Taxis, etc.) verschickt werden. Im Bereich der integrierten Versorgung existieren viele Modellprojekte unterschiedlicher Hersteller, ein allgemeingültiger Standard hieraus ist jedoch noch nicht etabliert. Das Themengebiet Health Information Exchange (HIE) spielt in diesem Zusammenhang zukünftig eine sehr wichtige Rolle und bietet neuartige Entwicklungsmöglichkeiten. Um die beschriebenen lebensbedrohlichen Situationen aufgrund falscher, fehlender oder zu spät gelieferter Patienteninformation zu vermeiden, muss gewährleistet werden, dass die notwendigen Informationen möglichst unkompliziert zur rechten Zeit, am richtigen Ort bereit gestellt werden.

Ein weiterer potenzieller Bereich für disruptive Sprünge bietet sich auch in der klinischen Entscheidungsunterstützung (z.B. in Anlehnung an IBM Watson, etc.). In diesem Zusammenhang spielen die Themen Big Data und Datenanalyse zukünftig eine 
wichtige Rolle. Die aggregierten gesundheitsbezogenen Daten sind für die Forschung von großer Bedeutung und implizieren u.a. eine anonymisierte und pseudonymisierte Auswertung von Sekundärdaten im Gesundheitswesen. Die IHE Public Health Domain beschäftigt sich mit standardisierten Verfahren, die es ermöglichen unter Einhaltung gesetzlicher Bestimmungen elektronisch auf die Daten zuzugreifen.

Konträr zum medizinischen Bereich bietet sich im Public Health Bereich, ein offenes weites Feld für neuartige bzw. disruptive Entwicklungen. Hier zeichnet sich ein vielmehr selbstbestimmtes Individuum ab, das sich mit dem eigenen Gesundheitszustand auseinandersetzt und dementsprechend andere Informationstechnologien außerhalb der Pathogenese einsetzt. Das Bewusstsein über die Wichtigkeit der Aufrechterhaltung oder Stärkung der eigenen Gesundheit ist stark ausgeprägt. Die hohe Selbstbestimmtheit in diesem Bereich führt zum Einsatz völlig neuartiger Techniken oder Informationstechnologien zur Kontrolle und Überwachung des eigenen Gesundheitszustandes. Im Vergleich zum medizinischen Bereich sind viele dieser Technologien nicht zwangsläufig an Standards oder Richtlinien gebunden, die erfüllt werden müssen. Als Beispiele hierfür können die sogenannten Wearables zur Überwachung von Vitalparametern, die in der Gesundheitsförderung und -prävention eingesetzt werden. Zudem kann allgemein das Internet als Quelle zur Informationseinholung und Austausch von Erfahrungen über Gesundheit und Krankheit mit allen Vorteilen und Risiken herangezogen werden. Das Thema der informierten Patienten oder auch sogenannten e-Patienten beherbergen in Gänze neue Anforderungen und Herausforderungen hinsichtlich der medizinischen Dokumentation und Aufklärung im pathogenetischen Bereich. Weitere Beispiele sind Gesundheitsportale, die Informationen und Schulungsprogramme in den Bereichen E-Mental-Health, betriebliches Gesundheitsmanagement etc. anbieten. Andere Gesundheitssysteme (wie z.B. USA, Australien etc.) haben in Gänze ein anderes Verständnis oder Auffassungen über die Themen Datenschutz und Datensicherheit. Vor diesem Hintergrund gibt es aus dem Ausland, insbesondere ausgehend und von den Global Playern (z.B. Google, Apple, etc.) Entwicklungen und Forschungen in Richtung Personal Health Record (PHR).

\section{Chancen und Risiken}

Die beschriebenen technologischen Entwicklungen in den Bereichen Public Health und Medizin funktionieren a ktuell autark und weitgehend unabhängig voneinander. Die Daten die im privaten Umfeld generiert werden, werden in einem Behandlungsverlauf im medizinischen Bereich in der Regel nicht herangezogen. Andersherum stehen dem Patienten die Daten aus dem medizinischen Bereich lediglich rudimentär zu Verfügung. Eine Vernetzung sowie zentrale Speicherung der Daten beider Bereiche, würde in sich viele Potenziale und Möglichkeiten eröffnen, die Gesundheitsversorgung zu verbessern.

\section{Chancen}

- historische Speicherung des Gesundheitszustandes an einem zentralen Speicher

- Einbindung eigener Gesundheitsdaten, z.B. aus dem Bereich der Gesundheitsförderung und -prävention 
- hohe Patientenselbstbestimmung durch Verwaltung und Zugriff auf eigene Gesundheitsdaten

- Möglichkeiten zur ganzheitlichen Analyse von Krankheitsbildern in der Bevölkerung

v übergreifende, klinische Entscheidungsunterstützung durch gezielte Bereitstellung von Informationen durch die IT zu einem Behandlungsfall

- bessere Kontrolle und Überwachung von Behandlungscodierungen und Abrechnung

- Chance zur Einbindung von Gesundheitsdaten aus dem Public Health Bereich

- Chance zur standardisierten und besseren Dokumentation hinsichtlich der Patientenaufklärung und des Behandlungsverlaufes

\section{Risiken}

- Datenschutz und Datensicherheit

- Missbrauch von Daten (z.B. werbliche Kriterien u.a.)

- Gefahr von Konfliktsituationen hinsichtlich der Kompetenzen durch den informierten Patienten, verbunden mit unklaren Haftungssituationen

- Zeitaufwand bei knappen Ressourcen

Zu den aufgeführten Chancen und Risiken eröffnen die neuen Technologien in Gänze komplett neue Umgangsformen und Rollenverständnisse. Im Rahmen der Erbringung von Gesundheitsleistungen liegt im medizinischen Bereich generell eine Mensch-zu-Mensch-Beziehung vor, die oft auf Vertrauen basiert. Dem Patienten obliegt dabei eine passive Rolle. Im Public Health Bereich liegt eine Mensch-zur-Maschine-Beziehung vor. Dem Individuum obliegt eine aktive Rolle hinsichtlich seiner Gesundheitsbelange. Beide Beziehungsformen beherbergen in sich eine Reihe von Chancen aber auch Risiken.

\section{Trends und Entwicklung}

Die beschriebenen Informationstechnologien in den Bereichen der Medizin und Public Health funktionieren derzeit parallel und unabhängig voneinander. Um entsprechende Potenziale, die sich aus beiden Themengebieten ergeben auszuschöpfen, ist langfristig eine Vernetzung beider Bereiche erforderlich. Ein langfristiger Trendverlauf wäre beispielsweise die Fusionierung.

Im medizinischen Bereich zeichnen sich folgende Trends und Entwicklungen der Technologien ab:

- ganzheitliche medizinische Dokumentenarchivierung und -management (Healthcare Content Management)

- Das PACS aus dem Bildgebungsbereich (z.B. Radiologie/Kardiologie) hat sich in der letzten Dekade zunehmend auf die Archivierung von Dokumenten auch außerhalb der Bildgebung spezialisiert. Die klassische Rolle des PACS, welches Bilddaten oder Befunde innerhalb der Radiologie oder Kardiologie archiviert, ist längst nicht mehr aktuell. Mittlerweile kann das System krankenhausweit auch Dokumente aus der Signalverarbeitung (z.B. EKGs) oder andere Dokumente aus der Patientenakte archivieren. 
Krankenhausweit entwickelt sich dieses System zu einem ganzheitlichen medizinischen Archiv, welches sämtliche medizinischen Daten standardkonform archiviert und zentral für unterschiedliche Anwendungen bereitstellt. Das medizinische Personal kann so, einfach und unkompliziert auf sämtliche Dokumente des Patienten digital zugreifen.

- Health Information Exchange und Interoperabilität

v Im Gesundheitswesen gibt es eine Reihe an Anbietern von IT-Systemen. Dem Thema der standardkonformen und interoperablen Schnittstellenumsetzung sollte ein besonderer Stellenwert beigemessen werden. Dabei sollte dieses Themengebiet gesondert von anderen Anwendungssystemen, wie z.B. die Archivierung, Bildverarbeitung, Dokumentenmanagement etc. bearbeitet werden. Dabei sollte die Idee von zentralisierten Informationsstrukturen gestärkt werden, welche ausschließlich die standardkonforme und interoperable Schnittstellenimplementierung, Datenkommunikation und -transport regeln und steuern.

- klinische Entscheidungsunterstützung

- Großes Entwicklungspotenzial beherbergt das Themengebiet der klinischen Entscheidungsunterstützung. Im medizinischen Bereich wäre das ein Kandidat für einen disruptiven Fortschritt bzw. Entwicklungssprung. Hierbei bilden die Themen Datenanalytik, Big Data und Wissensmanagement den nächsten Meilenstein, um hieraus Erkenntnisse für den klinischen Nutzer zu gewinnen. Die Auswertung von Sekundärdaten ist sowohl für die Forschung als auch für die Überwachung der Gesundheit in der Bevölkerung (Public Health) von großer Bedeutung.

- Telemedizin

I Die Telemedizin bildet eine Schnittmenge von Technologien aus den Bereichen der Public Health und Medizin. Hierbei zeichnet sich ein Trend hinsichtlich der medizinischen Fernüberwachung von Patienten, wie beispielsweise der Fernaufzeichnung und -überwachung von Vitalparametern, Telekardiologie etc. Hierbei zeichnet sich eine Kommunikation über mobile Endgeräte zwischen den Patienten und Ärzten ab.

Trends entwickeln sich im Public Health Bereich in Richtung der PHR, EHR(Electronic Health Record), die von Global Playern vorangetrieben werden. In Deutschland scheitern diese an gesetzlichen Bestimmungen und der fehlenden Bereitschaft, eigene Gesundheitsdaten auf diesen Plattformen abzulegen. Weitere Entwicklungspotenziale liegen in den Bereichen der Patienteninformierung, Schulungsprogramme sowie Technologien zur Überwachung der eignen Gesundheit im Rahmen der Gesundheitsförderung und -prävention. In diesem Zusammenhang zeichnen sich große Wachstumsmärkte bei Lösungen in den Bereichen der Wireless Health, Tele Health, Mobile Health, Automotive Health u.a. Gebiete ab. 